\title{
Optimal Control of ISTR Rumor Propagation Model with Social Reinforcement in Heterogeneous Network
}

\author{
Liang'an Huo $\mathbb{D D}^{1}$ Sijing Chen, ${ }^{1}$ Xiaoxiao Xie, ${ }^{1}$ Huiyuan Liu $\mathbb{D}^{2},{ }^{2}$ and Jianjia He ${ }^{1}$ \\ ${ }^{1}$ Business School, University of Shanghai for Science and Technology, Shanghai 200093, China \\ ${ }^{2}$ School of Statistics and Mathematics, Shanghai Lixin University of Accounting and Finance, Shanghai 201209, China \\ Correspondence should be addressed to Huiyuan Liu; lhysjtu@126.com
}

Received 14 September 2021; Revised 7 November 2021; Accepted 23 November 2021; Published 22 December 2021

Academic Editor: Xuzhen Zhu

Copyright ( 2021 Liang'an Huo et al. This is an open access article distributed under the Creative Commons Attribution License, which permits unrestricted use, distribution, and reproduction in any medium, provided the original work is properly cited.

\begin{abstract}
The wide spread of rumor is undoubtedly harmful to social stability; we should try to lower the effect of rumor on society. Therefore, it is reasonable to put forward the rumor control strategy on the basis of the study of the law of rumor propagation. Firstly, the ISTR model of rumor is established by including influencing factors of true information spreader and social reinforcement. And by using the next generation matrix method, the basic reproduction number of rumor is obtained. Then, in order to minimize the adverse effects of rumors, through introducing two control strategies of scientific knowledge popularization and refutation of rumors, the optimal control problem is established. And through using Pontryagin's Minimum Principle, the optimal solution of the rumor propagation model is solved. Finally, through theoretical analysis and numerical simulation, some results can be obtained. The results show that adding true information spreaders into the rumor model can effectively control the rumor propagation, and social reinforcement plays a significance role in rumor. The results also prove that these two control strategies can effectively inhibit the propagation of rumors. With the addition of control strategies, the number of true information spreaders increases, while the number of rumor spreaders decreases.
\end{abstract}

\section{Introduction}

In modern society, means of communication have developed very rapidly, and the spread of rumors basically relies on the new media of social networks. The flexibility of social media has greatly accelerated the speed of rumor propagating, which has exerted a great influence on People's Daily life [1]. For example, the incident of COVID-19 gave rise to many rumors, which were propagated among people through different channels. For example, there is a rumor that novel Coronavirus can be killed by drinking high concentration of alcohol or disinfection solution. Such a rumor sounds ridiculous, but still some people believe it, play jokes on their lives, and finally cause irreversible consequences. The rumors may affect people's judgment of events, make some absurd behaviors, seriously affect people's lives, and cause negative social effects. Therefore, studying the rumor propagation dynamics can significantly lower the adverse effects of rumor, so as to better control rumor propagation [2].
The rumor was first put forward by Daley and Kendall in the 1960s; they came up with rumor propagation DK model, which has become the basis of rumor propagation model research [3]. Then, based on the DK model, many excellent rumor propagation models such as the MK model and other variants were produced [4]. However, these models are based on the simple network structure, without considering the complex topology of network [5]. In real life, social networks have a highly complex topological structure, and rumor propagation channels and ways are very diverse. Therefore, with the progress of complex network theory, more attention has been paid to the theory of rumor propagation dynamics in complex network [6]. A deterministic MK model is set up by Zanette in small world networks [7]. Moreno et al. found out the numerical solution of the mean field equation and conducted simulation experiments to study the random version of the MK model in the scale-free network [8]. In addition, many human elements can also influence the rumor propagating process. 
Sun established an uncertain SIR (spreader, ignorant, stifler) rumor propagation model driven by a Liu process and studied the influence of disturbance on rumor propagation mechanism [9]. Wang et al. came up with a susceptibility removal model, in which confidence mechanism was introduced [10]. Zhao et al. built the model by considering the forgetting rate during rumor propagation [11]. Jiang proposed a novel rumor spreading-debunking (RSD) model by ordinary differential equation (ODE) system to explore the interplay mechanism between rumor spreading and debunking processes [12]. Wang introduced nonlinear suppression mechanism and established an IS2R2 model, which considers cross propagation in multilingual environment [13]. In order to explore the law of rumor propagation on online social networks and formulate corresponding control strategies, Yu proposed 2I2SR rumor propagation model with and without delay based on multilingual environment [14]. In homogeneous and inhomogeneous networks, Zhu et al. took into account forced silence functions and time delay, and then they proposed a new propagation model about rumor [15]. In addition, there are many different rumor propagation models, such as the SIR model [16], the SEIR model [17], the ILSR model [18], the SPNR model [19], the SAIR model [20], the SIR-UA model [21], and so on.

In addition, scholars have also studied many other factors that influence rumor propagation, such as social reinforcement. The definition of social reinforcement is that a person receives a great deal of information from the external environment before making a judgment or an action. Previous studies have shown that social reinforcement can affect individual communication behaviors and thus exert a significant influence on rumor propagation [22, 23]. When the rumor begins to propagate, individuals will be affected by multiple information from the outside world [24-26]; these social reinforcing effects can be applied to rumors as well as to other aspects. Huo et al. found that social reinforcement plays a significant role in rumor propagating through their research [27].

True information is also an important factor affecting the rumor propagation. True information is the truth of the rumors released by the relevant authorities in an emergency. There are many studies of true information. Vosoughi et al. investigated the differential spreading between all true and false news stories, which were confirmed and posted on Twitter from 2006 to 2017 [28]. Yang et al. came up with a competitive diffusion model to curb rumors by propagating true information on social networks [29]. Koohikamali uses model research to distinguish between rumor and fact [30]. In homogeneous networks, Zhao et al. further summarized the content of rumor dynamics after considering the refutation behavior [31]. When the rumors started to propagate, some people may know the true information about the rumor and spread the true information under social reinforcement. These people are called true information spreader. Therefore, it is more suitable to add true information spreader into the rumor model.

In addition to studying the rumor propagation dynamics, the research on rumor control can better weaken the adverse impact of rumor on the society. There have been many previous studies on rumor control. Through the calculation and analysis of the equilibrium point and basic reproduction number of the rumor model, Zhao et al. proposed that the information released by the government and network monitoring can better prevent the propagating of rumors [32]. By studying the influence of determination on rumor propagation, Askarizade et al. proposed an antirumor control mechanism [33]. Chen proposed a rumor propagation model to analyse the problem of rumor control in exigence [34]. Ahsan et al. demonstrated the efforts and achievements made so far in combating the spread of rumor information [35]. Huo et al. proposed the optimal control strategy and verified that it could minimize the amount of rumor spreaders and achieve an effective way of maximizing social utility [36].

In the above literatures, some methods to control rumors are discussed. In terms of controlling rumors, the two methods of popularizing knowledge to the ignorant and refuting rumors specifically to rumor spreaders can well control rumors. However, these two methods have rarely been considered in the previous literature, so this paper introduces two control strategies of scientific knowledge popularization and refutation of rumors to study the control of rumors. Among them, scientific knowledge popularization means promoting scientific knowledge to some people who have never heard of rumors and improving their scientific literacy. Refutation of rumors means refuting a rumor specifically against the person who hears the rumor. In this paper, all studies and analyses are based on heterogeneous networks. First, we consider the impact of social reinforcement and true information on rumor propagation dynamics and establish a rumor propagation ISTR model. Then, the problem of optimal rumor control is proposed and the optimal strategy of rumor control is studied based on the model. The main content of this text includes the following: The second part describes that, under the influence of social reinforcement, true information spreaders are added to the ISR model of rumor propagation and an ISTR model describing the rumor propagation dynamics. Then, a mean field method is used to calculate the average transition probability of different groups, and the mean field equation of the model is calculated. In the third part, the basic reproduction number is calculated in the heterogeneous network. In the fourth part, the rumor control model is established. Optimal control theory is applied and Pontryagin's principle is used to obtain the optimum result of control variables. In the fifth part, the simulation is used to verify the model and compare the changes of the model with or without control. The sixth part is conclusion.

\section{The ISTR Model}

In terms of the rumor propagation process, we consider that the group is composed of $N$ individuals, assuming that the rumor propagating is carried out through the direct contact between the spreader and others in the group. The connections between these groups occur in an undirected network of social interactions $G=(V, E)$, in which $V$ 
represents vertices and $E$ represents edges. In this model, $V$ denotes each individual, and $E$ denotes the connections between individuals.

All nodes are classified into four types: ignorant $(I)$, rumor spreader $(S)$, true information spreader $(T)$, and stifler $(R)$. Ignorant people are those who never heard rumor. Rumor spreaders propagate the rumor to the neighbors and do not know the true information about the rumor. True information spreaders grasp the true information about the rumor and choose to propagate the true information. Stiflers are those who stop propagating either rumor or true information. Based on this, we establish an ISTR model, and the assumptions of the model are as follows:

(1) Assuming this is an open system, then the ignorant will enter a particular community with coming rate $q$.

(2) It is assumed that, under the function of social reinforcement, the ignorant people will turn into rumor spreaders with probability $P_{I S}$ through the interaction with spreader neighbors. Similarly, when an ignorant person interacts with true information spreader neighbors, it is possible to become a true information spreader with probability $P_{I T}^{i}$.

(3) For the rumor spreader, when it contacts with the other one rumor spreader or a stifler, it has the potential to be a stifler in the probability $\alpha$. When the rumor spreader gets in touch with the true information spreader, it will become a true information spreader with probability $\beta$. Moreover, the rumor spreader is likely to be a stifler in the probability $\delta$.

(4) The rumor spreader and the true information spreader will become stiflers with probability $\delta$ under the influence of forgetting mechanism.

(5) Assume that people in all states will leave the community for some reason, which is called the leaving rate $d$.

The dynamic process of rumor propagation can be shown in Figure 1.

Human activities are complex, and individuals' actions and choices are always influenced by the surrounding environment. The researches have shown that a person is more likely to pick up the message and act on it when a person receives multiple signals from neighbors [20]. We call this effect social reinforcement. When rumors start to spread, the ignorant will be influenced by the rumor spreaders and the true information spreaders. Under the role of social reinforcement, if the number of times that the ignorant person hears a rumor increases, the likelihood that he believes the rumor increases, which can be called social reinforcement for rumor, denoted by $b$. If the number of times that the ignorant person hears the true information increases, the ignorant person is more likely to believe the true information, which can be called the social reinforcement for true information, denoted by $h$.

As shown in Figure 2, there are four groups around the ignorant node $i$, but it only interacts with rumor spreaders and true information spreaders. Suppose the ignorant node $i$ has $k$ neighbors, of which there are $f$ rumor spreaders and $g$ true information spreaders. In the absence of social reinforcement, when an ignorant person comes into contact with a rumor spreader, he or she will convert to rumor spreader with probability $\theta$. Similarly, in the absence of social reinforcement, when an ignorant person comes into contact with a true information spreader, he or she will change into a true information spreader in the probability $\eta$. Therefore, in the absence of social reinforcement, the probability of the ignorant remaining ignorant state is $1-\theta \Delta t$. On the contrary, under the effect of social reinforcement for rumor, rumors are more likely to propagate, and the probability that the ignorant still remains in ignorant state is $1-\theta(1+b) \Delta t$. When there are $f$ rumor spreaders around the ignorant node, the probability that the ignorant person remains in ignorant state is $[1-\theta(1+b) \Delta t]^{f}$, and then the probability that the ignorant node changes into the rumor spreader is $\left[1-(1-\theta(1+b) \Delta t)^{f}\right]$. Similarly, under the effect of social reinforcement for true information, when there are $g$ true information spreaders around the ignorant node, the probability that an ignorant person keeps the ignorant state is $[1-\eta(1+h) \Delta t]^{g}$, and the probability that the ignorant person transforms into true information spreader is $\left[1-(1-\eta(1+h) \Delta t)^{g}\right]$. However, ignorant people are affected by both rumor spreaders and true information spreaders, and then their state changes. Therefore, for ignorant node $i$, we denote with $P_{I S}^{i}$ the probability that ignorant becomes rumor spreader under time interval $[t, t+\Delta t] ;$ it then follows that

$$
P_{I S}^{i}=\left\{1-[1-\theta(1+b) \Delta t]^{f}\right\}[1-\eta(1+h) \Delta t]^{g} .
$$

Similarly, in the time interval $[t, t+\Delta t], P_{I T}^{i}$ denotes the possibility of ignorant node $i$ transforming into the true information spreader, and $P_{I I}^{i}$ means the possibility of ignorant node $i$ keeping the state of ignorant.

$$
\begin{aligned}
& P_{I T}^{i}=\left\{1-[1-\eta(1+h) \Delta t]^{g}\right\}[1-\theta(1+b) \Delta t]^{f}, \\
& P_{I I}^{i}=1-P_{I S}^{i}-P_{I T}^{i} .
\end{aligned}
$$

In the above formula, at time $t, f=f(t)$ denotes the amount of rumor spreaders around the ignorant node $i$, and the $g=g(t)$ denotes the amount of true information spreaders around the ignorant node $i$. Assuming that the ignorant node $i$ holds $k$ links, $f$ and $g$ are considered to be random variables and they follow the binomial distribution.

$$
\begin{aligned}
& \prod(f, t)=C_{k}^{f} \Theta_{1}^{f}(k, t)\left[1-\Theta_{1}(k, t)\right]^{k-f}, \\
& \prod(g, t)=C_{k-f}^{g} \Theta_{2}^{g}(k, t)\left[1-\Theta_{2}(k, t)\right]^{k-f-g} .
\end{aligned}
$$

At time $t, \Theta_{1}(k, t)$ means the probability that one side of the ignorant node of degree $k$ is connected to the rumor spreader with degree $k^{\prime}$, and $\Theta_{2}(k, t)$ indicates the probability that one side of the ignorant node of degree $k$ is connected to the true information spreader with degree $k^{\prime}$. The quantities are expressed as follows: 


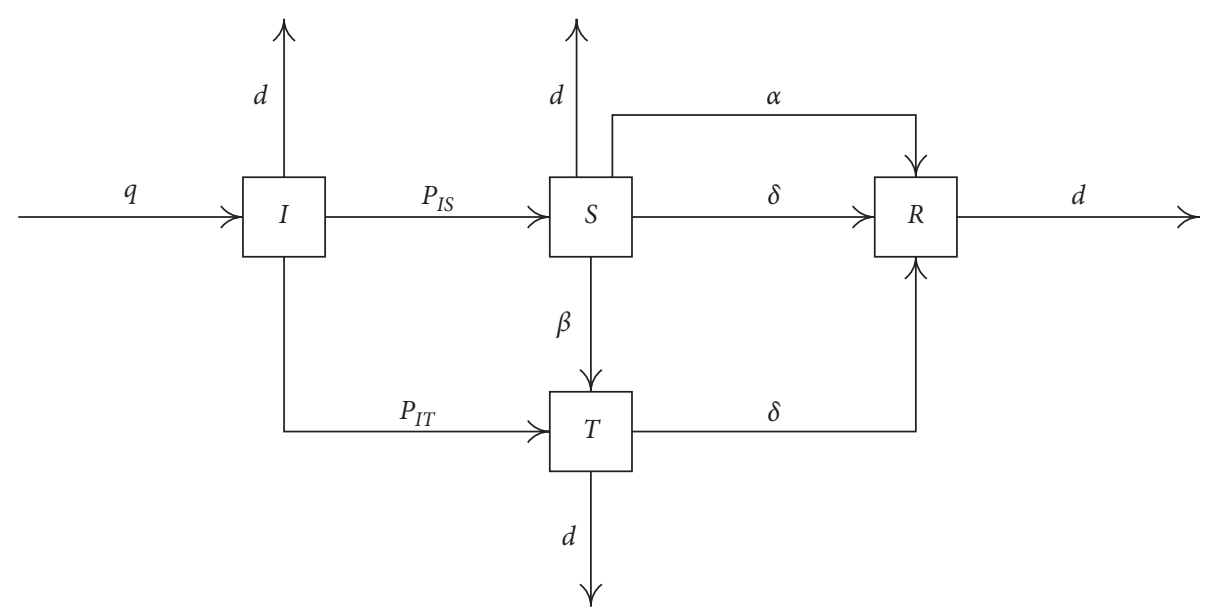

FIGURE 1: The dynamic process of rumor propagation.

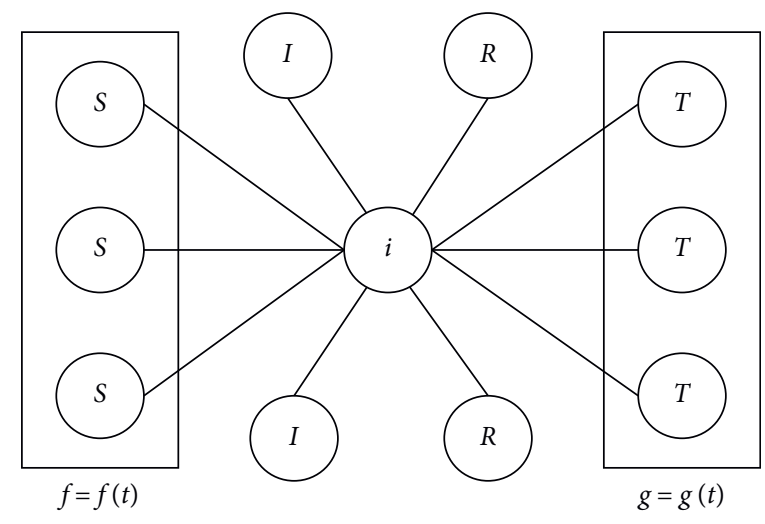

FIgURE 2: Ignorant node $i$ connects other nodes.

$$
\begin{aligned}
& \Theta_{1}(k, t)=\sum_{k^{\prime}} P\left(\frac{k^{\prime}}{k}\right) P\left(\frac{S_{k^{\prime}}}{I_{k}}\right) \approx \sum_{k^{\prime}} P\left(\frac{k^{\prime}}{k}\right) S_{k^{\prime}}(t), \\
& \Theta_{2}(k, t)=\sum_{k^{\prime}} P\left(\frac{k^{\prime}}{k}\right) P\left(\frac{T_{k^{\prime}}}{I_{k}}\right) \approx \sum_{k^{\prime}} P\left(\frac{k^{\prime}}{k}\right) T_{k^{\prime}}(t) .
\end{aligned}
$$

Among them, $P\left(k^{\prime} / k\right)$ means the degree correlation function. $P\left(S_{k^{\prime}} / I_{k}\right)$ indicates the contingent probability of the connection between the ignorant of degree $k$ and the rumor spreader of degree $k^{\prime}$, and $P\left(T_{k^{\prime}} / I_{k}\right)$ is conditional probability of the connection between the ignorant of degree $k$ and the true information spreader of degree $k^{\prime}$. In addition, $S_{k^{\prime}}(t)$ denotes the density of the node of rumor spreader at moment $t$, which belongs to the category of $k^{\prime}$. And $T_{k^{\prime}}(t)$ is the density of the node of true information spreader at moment $t$, which belongs to the category of $k^{\prime}$.

According to the above formula, in the time interval $[t, t+\Delta t]$, for ignorant nodes of degree $k$, we can get the average of the transition probability for all values of $f$ and $g$.

$$
\begin{aligned}
\bar{P}_{I S}(k, t)= & \sum_{f=0}^{k} \prod(f, t) \times \sum_{g=0}^{k-f} \prod(g, t) \times P_{I S}^{i} \\
= & \sum_{f=0}^{k} C_{k}^{f} \Theta_{1}^{f}(k, t)\left[1-\Theta_{1}(k, t)\right]^{k-f} \times \sum_{g=0}^{k-f} C_{k-f}^{g} \Theta_{2}^{g}(k, t)\left[1-\Theta_{2}(k, t)\right]^{k-f-g} \times P_{I S}^{i} \\
= & \left\{1-\eta(1+h) \Theta_{2}(k, t)\left(1-\Theta_{1}(k, t) \Delta t\right\}^{k}\right. \\
& -\left\{1-\left[\theta(1+b) \Theta_{1}(k, t)+\eta(1+h) \Theta_{2}(k, t)\left(1-\Theta_{1}(k, t)\right] \Delta t\right\}^{k} .\right.
\end{aligned}
$$




$$
\begin{aligned}
\bar{P}_{I T}(k, t)= & \sum_{g=0}^{k} \prod(g, t) \times \sum_{f=0}^{k-g} \prod(f, t) \times P_{I T}^{i} \\
= & \sum_{g=0}^{k} C_{k}^{g} \Theta_{2}^{g}(k, t)\left[1-\Theta_{2}(k, t)\right]^{k-g} \times \sum_{f=0}^{k-g} C_{k-g}^{f} \Theta_{1}^{f}(k, t)\left[1-\Theta_{1}(k, t)\right]^{k-f} \times P_{I T}^{i} \\
= & \left\{1-\theta(1+b) \Theta_{1}(k, t)\left(1-\Theta_{2}(k, t) \Delta t\right\}^{k}\right. \\
& -\left\{1-\left[\theta(1+b) \Theta_{1}(k, t)\left(1-\Theta_{2}(k, t)+\eta(1+h) \Theta_{2}(k, t)\right] \Delta t\right\}^{k} .\right. \\
\bar{P}_{I I}(k, t)= & 1-\bar{P}_{I S}(k, t)-\bar{P}_{I T}(k, t) .
\end{aligned}
$$

Similarly, through calculation, within the time interval $[t, t+\Delta t]$, the average transition probability of the rumor spreaders, true information spreaders, and stiflers of degree $k$ can be obtained.

$$
\begin{aligned}
& \bar{P}_{S T}(k, t)=k \beta \sum_{k^{\prime}} P\left(\frac{k^{\prime}}{k}\right) T_{k^{\prime}}(t) \Delta t . \\
& \bar{P}_{S R}(k, t)=k \alpha \sum_{k^{\prime}} P\left(\frac{k^{\prime}}{k}\right)\left[S_{k^{\prime}}(t)+R_{k^{\prime}}(t)\right] \Delta t-\delta \Delta t . \\
& \bar{P}_{S S}(k, t)=1-\bar{P}_{S T}(k, t)-\bar{P}_{S R}(k, t)-d \Delta t .
\end{aligned}
$$

$$
\bar{P}_{T R}(k, t)=\delta \Delta t .
$$

At time $t, I(k, t), S(k, t), T(k, t)$, and $R(k, t)$, respectively, represent the average quantity of ignorant, rumor spreaders, true information spreaders, and stiflers with degree $k$. According to equations (8)-(14), we can gain the average transition rate for the amount of all nodes with degree $k$ over the time interval $[t, t+\Delta t]$.

$$
\begin{aligned}
I(k, t+\Delta t) & \left.\left.=I(k, t)+q-I(k, t) \bar{P}_{I S}(k, t)\right)-I(k, t) \bar{P}_{I T}(k, t)\right)-I(k, t) d \Delta t . \\
S(k, t+\Delta t) & =S(k, t)+I(k, t) \bar{P}_{I S}(k, t)-S(k, t) \bar{P}_{S T}(k, t)-S(k, t) \bar{P}_{I R}(k, t)-S(k, t) d \Delta t . \\
T(k, t+\Delta t) & =T(k, t)+I(k, t) \bar{P}_{I T}(k, t)+S(k, t) \bar{P}_{S T}(k, t)-T(k, t) \bar{P}_{T R}(k, t)-T(k, t) d \Delta t . \\
R(k, t+\Delta t) & =R(k, t)+S(k, t) \bar{P}_{S R}(k, t)+T(k, t) \bar{P}_{T R}(k, t)-R(k, t) d \Delta t .
\end{aligned}
$$

At time $t$, we use $I_{k}(t), S_{k}(t), T_{k}(t)$, and $R_{k}(t)$ to indicate the density of ignorant, rumor spreader, true information spreader, and stifler in terms of degree $k$, respectively. These quantities satisfy the normalization condition $I_{k}(t)+S_{k}(t)+T_{k}(t)+R_{k}(t)=1$. According to equations (15)-(18), in the limit $\Delta t \longrightarrow 0$ we can get 


$$
\left\{\begin{array}{l}
\frac{\mathrm{dI}_{\mathrm{k}}(t)}{\mathrm{dt}}=q-k I_{k}(t)\left[\theta(1+b) \frac{1}{\langle k\rangle} \sum_{k^{\prime}} k^{\prime} P\left(k^{\prime}\right) S_{k^{\prime}}(t)+\eta(1+h) \frac{1}{\langle k\rangle} \sum_{k^{\prime}} k^{\prime} P\left(k^{\prime}\right) T_{k^{\prime}}(t)\right]-\mathrm{dI}_{\mathrm{k}}(t) \\
\frac{\mathrm{d} S_{\mathrm{k}}(t)}{\mathrm{dt}}=k I_{k}(t) \theta(1+b) \frac{1}{\langle k\rangle} \sum_{k^{\prime}} k^{\prime} P\left(k^{\prime}\right) S_{k^{\prime}}(t)-k S_{k}(t) \alpha \frac{1}{\langle k\rangle} \sum_{k^{\prime}} k^{\prime} P\left(k^{\prime}\right)\left(S_{k^{\prime}}(t)+R_{k^{\prime}}(t)\right) \\
-k S_{k}(t) \beta \frac{1}{\langle k\rangle} \sum_{k^{\prime}} k^{\prime} P\left(k^{\prime}\right) T_{k^{\prime}}(t)-\delta S_{k}(t)-\mathrm{d}_{\mathrm{k}}(t) \\
\frac{\mathrm{dT}_{\mathrm{k}}(t)}{\mathrm{dt}}=k I_{k}(t) \eta(1+h) \frac{1}{\langle k\rangle} \sum_{k^{\prime}} k^{\prime} P\left(k^{\prime}\right) T_{k^{\prime}}(t)+k S_{k}(t) \beta \frac{1}{\langle k\rangle} \sum_{k^{\prime}} k^{\prime} P\left(k^{\prime}\right) T_{k^{\prime}}(t)-\delta T_{k}(t)-\mathrm{dT}_{\mathrm{k}}(t) \\
\frac{\mathrm{dR}_{\mathrm{k}}(t)}{\mathrm{dt}}=k S_{k}(t) \alpha \frac{1}{\langle k\rangle} \sum_{k^{\prime}} k^{\prime} P\left(k^{\prime}\right)\left(S_{k^{\prime}}(t)+R_{k^{\prime}}(t)\right)+\delta S_{k}(t)+\delta T_{k}(t)-\mathrm{dR}_{\mathrm{k}}(t) .
\end{array}\right.
$$

\section{Basic Reproduction Number}

Theorem 1. Considering model (19), the following assertions can be obtained.

(1) There is always a rumor free equilibrium, $E^{0}=\left(I_{1}^{0}(t)\right.$, $\left.0,0,0, \cdots, I_{k}^{0}(t), 0,0,0\right)$, where $I_{k}^{0}(t)=q / d, 1 \leq k \leq n$.
(2) When $R_{0}=\rho\left(F V^{-1}\right)=q /(\delta+d) d \cdot\left\langle k^{2}\right\rangle /\langle k\rangle \max$ $\{\theta(1+b), \eta(1+h)\}$, if $R_{0}>1$, we can get $E^{*}=\left(I_{1}^{*}(t), S_{1}^{*}(t), T_{1}^{*}(t), R_{1}^{*}(t), \cdots, I_{k}^{*}(t), S_{k}^{*}(t), T_{k}^{*}\right.$ $\left.(t), R_{k}^{*}(t), \cdots, I_{n}^{*}(t), S_{n}^{*}(t), T_{n}^{*}(t), R_{n}^{*}(t)\right)$, this is a unique rumor equilibrium, and

$$
\begin{aligned}
I_{k}^{*}(t) & =\frac{q}{d+k\left[\theta(1+b) \Theta_{1}+\eta(1+h) \Theta_{2}\right]} \\
S_{k}^{*}(t) & =\frac{k \theta(1+b) \Theta_{1} \cdot q}{(k \alpha \psi+k \beta \phi+\delta+d)\left\{d+k\left[\theta(1+b) \Theta_{1}+\eta(1+h) \Theta_{2}\right]\right\}} \\
T_{k}^{*}(t) & =\frac{k \beta \phi \cdot k \theta(1+b) \Theta_{1} \cdot q+k \eta(1+h) \Theta_{2}(k \alpha \psi+k \beta \phi+\delta+d) \cdot q}{(\delta+d)(k \alpha \psi+k \beta \phi+\delta+d)\left\{d+k\left[\theta(1+b) \Theta_{1}+\eta(1+h) \Theta_{2}\right]\right\}} \\
R_{k}^{*}(t) & =\frac{k \theta(1+b) \Theta_{1}[(k \alpha \psi+\delta)(\delta+d)+k \beta \phi \delta] \cdot q+k \eta(1+h) \Theta_{2}(k \alpha \psi+k \beta \phi+\delta+d) \cdot q}{d(\delta+d)(k \alpha \psi+k \beta \phi+\delta+d)\left\{d+k\left[\theta(1+b) \Theta_{1}+(1+h) \Theta_{2}\right]\right\}}, 1 \leq k \leq n
\end{aligned}
$$

Proof. For model (19), through calculating, it is easily verified that there exists an obvious equilibrium point
$E^{0}=\left(I_{1}^{0}(t), 0,0,0, \cdots, I_{k}^{0}(t), 0,0,0\right)$, where $I_{k}^{0}(t)=q / d$; then claim (1) is proved.

When $S_{k}(t) \neq 0,1 \leq k \leq n$, we get

$$
\begin{aligned}
I_{k}^{*}(t) & =\frac{q}{d+k\left[\theta(1+b) \Theta_{1}+\eta(1+h) \Theta_{2}\right]}, \\
S_{k}^{*}(t) & =\frac{k \theta(1+b) \Theta_{1} \cdot q}{(k \alpha \psi+k \beta \phi+\delta+d)\left\{d+k\left[\theta(1+b) \Theta_{1}+\eta(1+h) \Theta_{2}\right]\right\}}, \\
T_{k}^{*}(t) & =\frac{k \beta \phi \cdot k \theta(1+b) \Theta_{1} \cdot q+k \eta(1+h) \Theta_{2}(k \alpha \psi+k \beta \phi+\delta+d) \cdot q}{(\delta+d)(k \alpha \psi+k \beta \phi+\delta+d)\left\{d+k\left[\theta(1+b) \Theta_{1}+\eta(1+h) \Theta_{2}\right]\right\}}, \\
R_{k}^{*}(t) & =\frac{k \theta(1+b) \Theta_{1}[(k \alpha \psi+\delta)(\delta+d)+k \beta \phi \delta] \cdot q+k \eta(1+h) \Theta_{2}(k \alpha \psi+k \beta \phi+\delta+d) \cdot q}{d(\delta+d)(k \alpha \psi+k \beta \phi+\delta+d)\left\{d+k\left[\theta(1+b) \Theta_{1}+(1+h) \Theta_{2}\right]\right\}}, 1 \leq k \leq n
\end{aligned}
$$


$\Theta_{1}=1 /\langle k\rangle \sum_{k^{\prime}} k^{\prime} P\left(k^{\prime}\right) S_{k^{\prime}}(t), \quad \Theta_{2}=1 /\langle k\rangle \sum_{k^{\prime}} k^{\prime} P\left(k^{\prime}\right)$ $T_{k^{\prime}}(t), \quad \psi=1 /\langle k\rangle \sum_{k^{\prime}} k^{\prime} P\left(k^{\prime}\right)\left(S_{k^{\prime}}(t)+R_{k^{\prime}}(t)\right), \quad \phi=1 /\langle k\rangle$ $\sum_{k^{\prime}} k^{\prime} P\left(k^{\prime}\right) T_{k^{\prime}}(t)$. We let $x=\left(S_{k}(t), T_{k}(t), I_{k}(t), R_{k}(t)\right)$; then model (19) can be written as $\mathrm{dx} / \mathrm{dt}=\mathscr{F}(x)-v(x)$, where

$$
\mathscr{F}(x)=\left(\begin{array}{c}
k I_{k}(t) \theta(1+b) \frac{1}{\langle k\rangle} \sum_{k^{\prime}} k^{\prime} P\left(k^{\prime}\right) S_{k^{\prime}}(t) \\
k I_{k}(t) \eta(1+h) \frac{1}{\langle k\rangle} \sum_{k^{\prime}} k^{\prime} P\left(k^{\prime}\right) T_{k^{\prime}}(t) \\
0
\end{array}\right),
$$

When $E^{0}=(q / d, 0,0,0, \cdots, q / d, 0,0,0)$, the Jacobian matrices of $\mathscr{F}(x)$ and $\nu(x)$ are

$$
\mathrm{DF}\left(E_{0}\right)=\left(\begin{array}{cc}
F & 0 \\
0 & 0
\end{array}\right), \mathrm{D} v\left(E_{0}\right)=\left(\begin{array}{ll}
V & 0 \\
J_{1} & J_{2}
\end{array}\right) .
$$

where $J_{1}, J_{2}$ is matrix that can be omitted because it does not work in the following calculations:

$$
\begin{aligned}
F & =\left(\begin{array}{cc}
k \theta(1+b) \frac{q}{d} \frac{\left\langle k^{2}\right\rangle}{\langle k\rangle} & 0 \\
0 & \eta(1+h) \frac{q}{d} \frac{\left\langle k^{2}\right\rangle}{\langle k\rangle}
\end{array}\right), \\
V & =\left(\begin{array}{cc}
\delta+d & 0 \\
0 & \delta+d
\end{array}\right),
\end{aligned}
$$

in which $\left\langle k^{2}\right\rangle=\sum_{k} k^{2} P(k)$. According to the concept of [1], the reproduction number of model (19) is defined by $R_{0}=\rho\left(F V^{-1}\right)$, where $\rho\left(F V^{-1}\right)$ is the spectral radius of the matrix $F V^{-1}$. By calculating, we get

$$
R_{0}=\rho\left(F V^{-1}\right)=\frac{q}{(\delta+d) d} \cdot \frac{\left\langle k^{2}\right\rangle}{\langle k\rangle} \max \{\theta(1+b), \eta(1+h)\} .
$$

It can be seen from equation (22) that $R_{0}=q /(\delta+d) d .\left\langle k^{2}\right\rangle /\langle k\rangle \eta(1+h)$. when $\theta(1+b)<\eta$ $(1+h)$, the spread of true information has an impact on the threshold of rumor spread. The basic reproduction number $R_{0}$ represents the final threshold of the rumor propagation model. According to [34], if $R_{0}>1$, model (2) reaches a unique rumor equilibrium $E^{*}$, and claim (2) is proved.

\section{The Optimal Control Strategies}

Based on the rumor propagation model (19), in order to effectively control the propagation of rumor, we adopt the optimal control theory to discuss the law of control of rumor. Through the above analysis, it is apparent that the amount of rumor spreaders greatly affects the efficiency of rumor propagation. Therefore, we can adopt some strategies to lessen the amount of rumor spreaders to control rumors. After analysis and discussion, we believe that the two 
methods of popularizing knowledge and refuting rumors can control rumors well. However, these two methods have rarely been considered in the previous literature, so this paper introduces two control strategies of scientific knowledge popularization and refutation of rumors to study the control of rumors. Among them, scientific knowledge popularization means promoting scientific knowledge to some people who have never heard of rumors and improving their scientific literacy. Refutation of rumors means refuting a rumor specifically against the person who hears the rumor.

First of all, we introduce two control variables, $u_{k}$ and $v_{k}$. On the one hand, for the ignorant, authorities often organize some popularization and lectures of science knowledge, which will increase the probability of ignorant people turning into true information spreaders. Therefore, under the influence of scientific knowledge popularization, the conversion ratio of ignorant people turning to true information spreaders per unit of time is $u_{k}$. On the other hand, as for rumor spreader, authorities can conduct special rumor refutation activities aimed at rumors, which will increase the probability of rumor spreaders turning into true information spreaders. Under the role of rumor refutation strategies, the conversion ratio of rumor spreaders turning to true information spreaders per unit of time is $v_{k}$. Here, $U=$ $\left\{\left(u_{k}, v_{k}\right) \mid 0 \leq u_{k} \leq 1 / \eta(1+h)-1,0 \leq v_{k} \leq 1 / \beta-1, t \in[0, T]\right.$ $k=1,2, \cdots, n\}$ is the admissible control, where $u_{k}(t)$, $v_{k}(t) \in U$.

If rumor propagation is not restrained, it will have a certain impact on the society. Therefore, for the sake of preferably lessening the harm of rumors, we ought to study the strategies to control rumors. In the rumor system, the rumor spreaders have a huge effect on the rumor propagating; a low amount of rumor spreaders can availably control rumor propagating. In other words, the goal of rumor control is to maintain the amount of rumor spreaders to a minimum in a group. Moreover, by adding the above two control strategies to the rumor propagation model, we can more effectively reduce the number of rumor spreaders. Therefore, we set up the following objective function:

$$
J(u(t), v(t))=\int_{0}^{T} \sum_{k=1}^{n}\left(S_{k}(t)+\frac{a_{k}}{2} u_{k}^{2}(t)+\frac{b_{k}}{2} v_{k}^{2}(t)\right) \mathrm{dt},
$$

subject to

$$
\left\{\begin{array}{l}
\frac{\mathrm{dI}_{\mathrm{k}}(t)}{\mathrm{dt}}=q-k I_{k}(t)\left[\theta(1+b) \frac{1}{\langle k\rangle} \sum_{k^{\prime}} k^{\prime} P\left(k^{\prime}\right) S_{k^{\prime}}(t)+\left(1+u_{k}(t)\right) \eta(1+h) \frac{1}{\langle k\rangle} \sum_{k^{\prime}} k^{\prime} P\left(k^{\prime}\right) T_{k^{\prime}}(t)\right]-\mathrm{dI}_{\mathrm{k}}(t) \\
\frac{\mathrm{d} S_{\mathrm{k}}(t)}{\mathrm{dt}}=k I_{k}(t) \theta(1+b) \frac{1}{\langle k\rangle} \sum_{k^{\prime}} k^{\prime} P\left(k^{\prime}\right) S_{k^{\prime}}(t)-k S_{k}(t) \alpha \frac{1}{\langle k\rangle} \sum_{k^{\prime}} k^{\prime} P\left(k^{\prime}\right)\left(S_{k^{\prime}}(t)+R_{k^{\prime}}(t)\right) \\
-\left(1+v_{k}(t)\right) k S_{k}(t) \beta \frac{1}{\langle k\rangle} \sum_{k^{\prime}} k^{\prime} P\left(k^{\prime}\right) T_{k^{\prime}}(t)-\delta S_{k}(t)-d S_{k}(t) \\
\frac{\mathrm{dT}_{\mathrm{k}}(t)}{\mathrm{dt}}=k I_{k}(t)\left(1+u_{k}(t)\right) \eta(1+h) \frac{1}{\langle k\rangle} \sum_{k^{\prime}} k^{\prime} P\left(k^{\prime}\right) T_{k^{\prime}}(t)-\delta T_{k}(t)-\mathrm{dT}_{\mathrm{k}}(t) \\
+\left(1+v_{k}(t)\right) k S_{k}(t) \beta \frac{1}{\langle k\rangle} \sum_{k^{\prime}} k^{\prime} P\left(k^{\prime}\right) T_{k^{\prime}}(t) \\
\frac{\mathrm{dR} \mathrm{R}_{\mathrm{k}}(t)}{\mathrm{dt}}=k S_{k}(t) \alpha \frac{1}{\langle k\rangle} \sum_{k^{\prime}} k^{\prime} P\left(k^{\prime}\right)\left(S_{k^{\prime}}(t)+R_{k^{\prime}}(t)\right)+\delta S_{k}(t)+\delta T_{k}(t)-\mathrm{dR}_{\mathrm{k}}(t) .
\end{array}\right.
$$


$a_{k}, b_{k}, k=1,2, \cdots, n$ are small positive constants. For the sake of figuring out the optimal control problems (27) and (28), we should find the following Lagrangian:

$$
L=\int_{0}^{T} \sum_{k=1}^{n}\left(S_{k}(t)+\frac{a_{k}}{2} u_{k}^{2}(t)+\frac{b_{k}}{2} v_{k}^{2}(t)\right) d t .
$$

Then we set the Hamiltonian of the control problem as follows, from which we can obtain the minimum of the objective functional:

$$
H=L+\sum_{k=1}^{n}\left[\lambda_{1 k}(t) \frac{d I_{k}(t)}{\mathrm{dt}}+\lambda_{2 k}(t) \frac{d S_{k}(t)}{\mathrm{dt}}+\lambda_{3 k}(t) \frac{d T_{k}(t)}{\mathrm{dt}}+\lambda_{4 k}(t) \frac{d R_{k}(t)}{\mathrm{dt}}\right] .
$$

$\lambda_{1 k}(t), \lambda_{2 k}(t), \lambda_{3 k}(t), \lambda_{4 k}(t), k=1,2, \cdots, n$ are the adjoint functions.

Theorem 2. For the optimal control problem $J\left(u^{*}(t), v^{*}(t)\right)=\min J(u(t), v(t))$, there are optimal control variables $u^{*}(t)=\left(u_{1}^{*}(t), u_{2}^{*}(t), \cdots u_{n}^{*}(t)\right)$ and $v^{*}(t)=\left(v_{1}^{*}(t), v_{2}^{*}(t), \cdots v_{n}^{*}(t)\right)$. Assume that $I^{*}(t), S^{*}(t)$,
$T^{*}(t), R^{*}(t)$ are the optimal state solutions for the control problem, where $I^{*}(t)=\left(I_{1}^{*}(t), I_{2}^{*}(t), \cdots, I_{n}^{*}(t)\right), S^{*}(t)=$ $\left(S_{1}^{*}(t), S_{2}^{*}(t), \cdots, S_{n}^{*}(t)\right), T^{*}(t)=\left(T_{1}^{*}(t), T_{2}^{*}(t), \cdots, T_{n}^{*}(t)\right)$, $R^{*}(t)=\left(R_{1}^{*}(t), R_{2}^{*}(t), \cdots, R_{n}^{*}(t)\right)$. And there are adjoint variables $\lambda_{1 k}(t), \lambda_{2 k}(t), \lambda_{3 k}(t), \lambda_{4 k}(t), k=1,2, \cdots, n$ satisfying the following equalities

$$
\begin{aligned}
\frac{\mathrm{d} \lambda_{1 \mathrm{k}}}{\mathrm{dt}}= & \left(\lambda_{1 k}(t)-\lambda_{2 k}(t)\right)\left[k \theta(1+b) \Theta_{1}^{*}+\left(\lambda_{1 k}(t)-\lambda_{3 k}(t)\right)\left[\left(1+u_{k}(t)\right) k \eta(1+h) \Theta_{2}^{*}\right]+\lambda_{1 k}(t) d,\right. \\
\frac{d \lambda_{2 k}}{d t}= & -1+\left(\lambda_{1 k}(t)-\lambda_{2 k}(t)\right) \theta(1+b) \frac{k^{2} P(k)}{\langle k\rangle} I_{k}^{*}+\left(\lambda_{2 k}(t)-\lambda_{3 k}(t)\right)\left(1+v_{k}(t)\right) k \beta \phi^{*} \\
& +\left(\lambda_{2 k}(t)-\lambda_{4 k}(t)\right)\left[k \alpha \psi^{*}+\alpha \frac{k^{2} P(k)}{\langle k\rangle} S_{k}^{*}+\delta\right]+\lambda_{2 k}(t) d, \\
\frac{d \lambda_{3 k}}{d t}= & \left(\lambda_{1 k}(t)-\lambda_{3 k}(t)\right)\left(1+u_{k}(t)\right) \eta(1+h) \frac{k^{2} P(k)}{\langle k\rangle} I_{k}^{*}+\lambda_{3 k}(t)(\delta+d) \\
& +\left(\lambda_{2 k}(t)-\lambda_{3 k}(t)\right)\left(1+v_{k}(t)\right) \beta \frac{k^{2} P(k)}{\langle k\rangle} S_{k}^{*}-\lambda_{4 k}(t) \delta \\
\frac{d \lambda_{4 k}}{d t}= & \left(\lambda_{2 k}(t)-\lambda_{4 k}(t)\right) \alpha \frac{k^{2} P(k)}{\langle k\rangle} S_{k}^{*}+\lambda_{4 k}(t) d,
\end{aligned}
$$

with transversality conditions

$$
\lambda_{1 k}(t)=\lambda_{2 k}(t)=\lambda_{3 k}(t)=\lambda_{4 k}(t)=0, k=1, \cdots, n
$$

Furthermore, the optimal control $u_{1}^{*}(t), u_{2}^{*}(t), \cdots u_{n}^{*}(t)$ and $v_{1}^{*}(t), v_{2}^{*}(t), \cdots v_{n}^{*}(t)$ is obtained by

$$
\begin{aligned}
& u_{k}^{*}=\max \left\{\min \left\{\frac{\lambda_{1 k}(t)-\lambda_{3 k}(t)}{a_{k}} k \eta(1+h) I_{k}^{*} \frac{1}{\langle k\rangle} \sum_{k^{\prime}} k^{\prime} P\left(k^{\prime}\right) T_{k}^{*}, \frac{1}{\eta(1+h)}\right\}, 0\right\}, \\
& v_{k}^{*}=\max \left\{\min \left\{\frac{\lambda_{2 k}(t)-\lambda_{3 k}(t)}{k} k \beta S_{k}^{*} \frac{1}{\langle k\rangle} \sum_{k^{\prime}} k^{\prime} P\left(k^{\prime}\right) T_{k}^{*}, \frac{1}{\beta}-1\right\}, 0\right\} .
\end{aligned}
$$


Proof. By using the Hamiltonian (13), we obtain the deterministic adjoint equation and the transversality conditions. And from the setting $I_{k}(t)=I_{k}^{*}(t)$, $S_{k}(t)=S_{k}^{*}(t), T_{k}(t)=T_{k}^{*}(t)$, we get

$$
\begin{aligned}
\frac{d \lambda_{1 k}}{d t}= & \left(\lambda_{1 k}(t)-\lambda_{2 k}(t)\right)\left[k \theta(1+b) \Theta_{1}^{*}+\left(\lambda_{1 k}(t)-\lambda_{3 k}(t)\right)\left[\left(1+u_{k}(t)\right) k \eta(1+h) \Theta_{2}^{*}\right]+\lambda_{1 k}(t) d\right. \\
\frac{d \lambda_{2 k}}{d t}= & -1+\left(\lambda_{1 k}(t)-\lambda_{2 k}(t)\right) \theta(1+b) \frac{k^{2} P(k)}{\langle k\rangle} I_{k}^{*}+\left(\lambda_{2 k}(t)-\lambda_{3 k}(t)\right)\left(1+v_{k}(t)\right) k \beta \phi^{*} \\
& +\left(\lambda_{2 k}(t)-\lambda_{4 k}(t)\right)\left[k \alpha \psi^{*}+\alpha \frac{k^{2} P(k)}{\langle k\rangle} S_{k}^{*}+\delta\right]+\lambda_{2 k}(t) d \\
\frac{d \lambda_{3 k}}{d t}= & \left(\lambda_{1 k}(t)-\lambda_{3 k}(t)\right)\left(1+u_{k}(t)\right) \eta(1+h) \frac{k^{2} P(k)}{\langle k\rangle} I_{k}^{*}+\lambda_{3 k}(t)(\delta+d) \\
& +\left(\lambda_{2 k}(t)-\lambda_{3 k}(t)\right)\left(1+v_{k}(t)\right) \beta \frac{k^{2} P(k)}{\langle k\rangle} S_{k}^{*}-\lambda_{4 k}(t) \delta \\
\frac{d \lambda_{4 k}}{d t}= & \left(\lambda_{2 k}(t)-\lambda_{4 k}(t)\right) \alpha \frac{k^{2} P(k)}{\langle k\rangle} S_{k}^{*}+\lambda_{4 k}(t) d .
\end{aligned}
$$

Under optimal conditions, we have

$$
\begin{gathered}
\frac{\partial H}{\partial u_{k}(t)}=a_{k} u_{k}^{*}(t)-\left(\lambda_{1 k}(t)-\lambda_{3 k}(t)\right) k \eta(1+h) I_{k}^{*} \frac{1}{\langle k\rangle} \sum_{k^{\prime}} k^{\prime} P\left(k^{\prime}\right) T_{k}^{*}=0, \\
\frac{\partial H}{\partial v_{k}(t)}=b_{k} v_{k}^{*}(t)-\left(\lambda_{2 k}(t)-\lambda_{3 k}(t)\right) k \beta S_{k}^{*} \frac{1}{\langle k\rangle} \sum_{k^{\prime}} k^{\prime} P\left(k^{\prime}\right) T_{k}^{*}=0 .
\end{gathered}
$$

It follows that

$$
\left\{\begin{array}{l}
u_{k}^{*}=\frac{\lambda_{1 k}(t)-\lambda_{3 k}(t)}{k} k \eta(1+h) I_{k}^{*} \frac{1}{\langle k\rangle} \sum_{k^{\prime}} k^{\prime} P\left(k^{\prime}\right) T_{k}^{*}, \\
v_{k}^{*}=\frac{\lambda_{2 k}(t)-\lambda_{3 k}(t)}{k} k \beta S_{k}^{*} \frac{1}{\langle k\rangle} \sum_{k^{\prime}} k^{\prime} P\left(k^{\prime}\right) T_{k}^{*} .
\end{array}\right.
$$


As regards the properties of the control set $U$, it can be obtained that

$$
\begin{aligned}
& \left\{\begin{array}{l}
u_{k}^{*}=0, i f \frac{\lambda_{1 k}(t)-\lambda_{3 k}(t)}{a_{k}} k(1+h) I_{k}^{*} \frac{1}{\langle k\rangle} \sum_{k^{\prime}} k^{\prime} P\left(k^{\prime}\right) S_{k}^{*} \leq 0 \\
0<u_{k}^{*} \leq \frac{1}{\eta(1+h)}, i f 0<\frac{\lambda_{1 k}(t)-\lambda_{3 k}(t)}{a_{k}} k(1+h) I_{k}^{*} \frac{1}{\langle k\rangle} \sum_{k^{\prime}} k^{\prime} P\left(k^{\prime}\right) S_{k}^{*}<\frac{1}{\eta(1+h)} \\
u_{k}^{*}=\frac{1}{\eta(1+h)}, i f \frac{\lambda_{1 k}(t)-\lambda_{3 k}(t)}{a_{k}} k(1+h) I_{k}^{*} \frac{1}{\langle k\rangle} \sum_{k^{\prime}} k^{\prime} P\left(k^{\prime}\right) S_{k}^{*} \geq \frac{1}{\eta(1+h)} \\
v_{k}^{*}=0, i f \frac{\left.\lambda_{2 k}(t)-\lambda_{3 k}(t)\right)}{b_{k}} k \beta S_{k}^{*} \frac{1}{\langle k\rangle} \sum_{k^{\prime}} k^{\prime} P\left(k^{\prime}\right) T_{k}^{*} \leq 0 \\
0<v_{k}^{*} \leq \frac{1}{\beta}, i f 0<\frac{\lambda_{2 k}(t)-\lambda_{3 k}(t)}{b_{k}^{2}} k \beta S_{k}^{*} \frac{1}{\langle k\rangle} \sum_{k^{\prime}} k^{\prime} P\left(k^{\prime}\right) T_{k}^{*}<\frac{1}{\beta} \\
v_{k}^{*}=\frac{1}{\beta}, i f \frac{\lambda_{2 k}(t)-\lambda_{3 k}(t)}{b_{k}^{2}} k \beta S_{k}^{*} \frac{1}{\langle k\rangle} \sum_{k^{\prime}} k^{\prime} P\left(k^{\prime}\right) T_{k}^{*} \geq \frac{1}{\beta}
\end{array}\right.
\end{aligned}
$$

By sorting out the above formula, the optimal control solution $u_{k}^{*}(t) v_{k}^{*}(t)$ is obtained as follows:

$$
\begin{aligned}
& u_{k}^{*}=\max \left\{\min \left\{\frac{\lambda_{1 k}(t)-\lambda_{3 k}(t)}{k} k \eta(1+h) I_{k}^{*} \frac{1}{\langle k\rangle} \sum_{k^{\prime}} k^{\prime} P\left(k^{\prime}\right) T_{k}^{*}, \frac{1}{\eta(1+h)}\right\}, 0\right\}, \\
& v_{k}^{*}=\max \left\{\min \left\{\frac{\lambda_{2 k}(t)-\lambda_{3 k}(t)}{k} k \beta S_{k}^{*} \frac{1}{\langle k\rangle} \sum_{k^{\prime}} k^{\prime} P\left(k^{\prime}\right) T_{k}^{*}, \frac{1}{\beta}\right\}, 0\right\} .
\end{aligned}
$$

The transversality conditions are $\lambda_{1 k}(t)=\lambda_{2 k}(t)=$ $\lambda_{3 k}(t)=\lambda_{4 k}(t)=0, k=1, \cdots, n$.

\section{Numerical Simulations}

This part verifies the previous theoretical research on scalefree networks through numerical simulation. The degree distribution is $P(k)=2 m^{2} k^{-3}$, in which $m=3$ is the minimum degree of the network. In following experiments, firstly, based on model (19), we simulated the condition without rumor control. We investigate the impact of various parameters on the density of rumor spreader and true information spreader without control. Then, based on model (28), we add the control variables $u$ and $v$ to the simulation, respectively, and analyse the change of the density ignorant, rumor spreader, true information spreader, and stifler with or without control.

Figure 3 displays the time evolution chart about the influence of various parameters on the density change of rumor spreader without control. From the picture, we can see that, at the beginning, the rumor spreader begins to propagate with an initial value, and with the evolution of time, the density of rumor spreader first rises to the peak and then decreases to 0 . Figure $3(\mathrm{a})$ is the effects of social reinforcement for rumor $b$ on the spreader. It shows that larger social reinforcement for rumor leads to larger number of rumor spreaders and makes it reach a higher peak. This is because larger social reinforcement for rumor indicates larger positive influence of the external society on the rumor. In other words, there is more information favorable to 


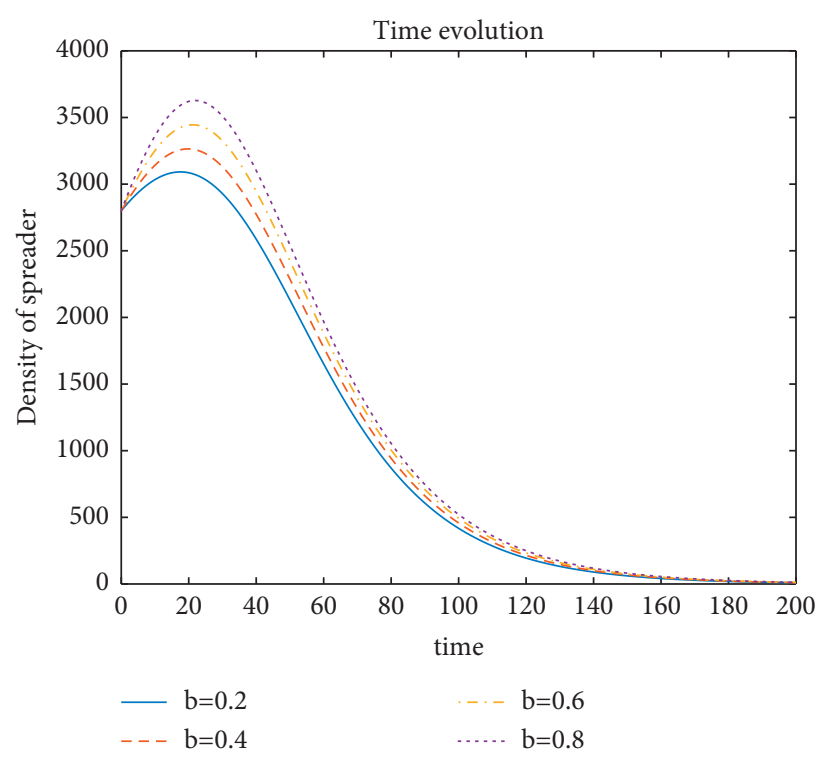

(a)

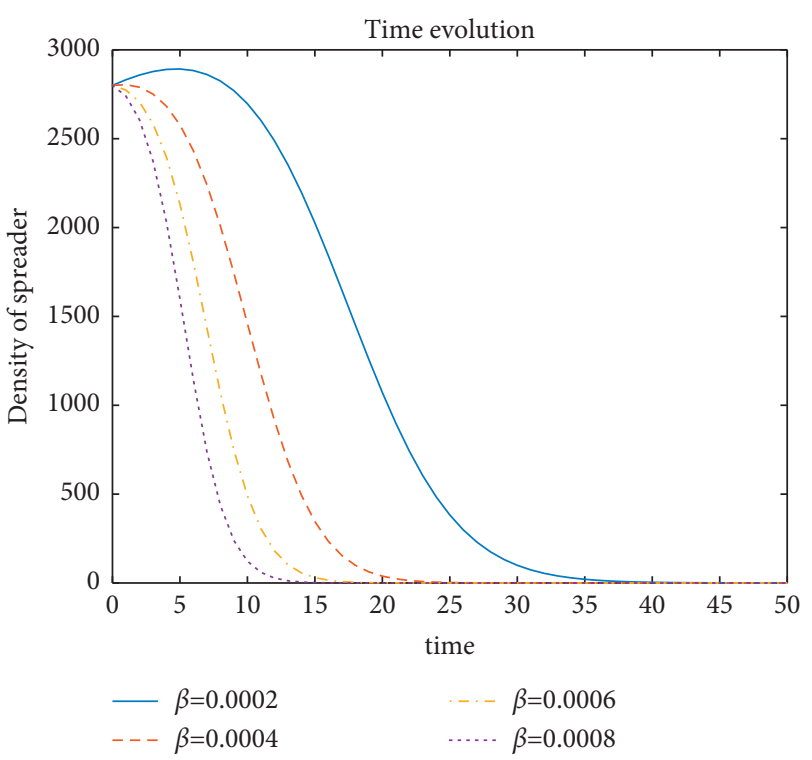

(b)

FIgURE 3: The impact of different parameters $b, \beta$ on the density of rumor spreader without control.

rumors in the society, and it is easier for people to believe rumors. As a result, ignorant people are more easily to be transformed into rumor spreaders, and the number of rumor spreaders will increase rapidly. In contrast, Figure 3(b) shows that smaller $\beta$ results in a larger number of rumor spreaders and a slower speed of declining to zero in the end. This is because this parameter indicates the transition of the rumor spreader to other states. Therefore, a smaller parameter leads to slower speed, thus reducing the number of rumor spreaders. In addition, it is obvious that the social reinforcement for rumor factor has a greater impact on the rumor spreader.

Figure 4 displays the change of the density of true information spreaders with time under different parameters without control. It is found that, at the beginning, there is no true information spreader in the system. As time goes on, the density of true information spreader first gradually increases to the maximum value and then decreases gradually. The parameters of the graphs in Figure 4 are social reinforcement for true information $h$ and true information conversion rate $\beta$. Social reinforcement for true information $h$ stands for true information about rumors. It can be seen from Figure 4(a) that greater $h$ results in higher rise rate and larger peak value of true information spreader. Larger social reinforcement for true information means that people are more likely not to believe rumors, and more ignorant people become true information spreader. Figure 4(b) shows that larger $\beta$ also makes the amount of true information spreaders get to a greater vertex at a faster speed. $\beta$ represents the probability that a rumor spreader changes into a true information spreader. In the society, when this value is larger, the rumor spreader is more likely to doubt the rumor and thus become the true information spreader.

Figure 5 is a comparison graph with or without control. Based on model (28), we add the control variables $u$ and $v$ to the simulation, respectively. There are four curves in each subgraph, respectively, representing the four control situations: (i) without control, (ii) with control variable $u$, (iii) with control variable, and (iv) with control variables $u$ and $v$. We find that the control variable $u$ has a greater influence on the density of true information spreader and ignorant. When the control variable $u$ is added, the density of true information spreader rises faster and the maximum density is higher. And the density of the ignorant declines faster in a smaller time. This is because the control variable $u$ is a control strategy added in the ignorant stage, making the ignorant true to the true information spreader, while the control variable $v$ is the control strategy added in the stage of the rumor spreader, making the rumor spreader become true information spreader. Therefore, the control variable $v$ has a greater influence on the density of the rumor spreader and true information spreader. With the addition of the control variable $v$, the density of the rumor spreader becomes smaller; it reaches a higher vertex faster and eventually reduces to a minimum in a shorter time. And as the density of true information spreader rises faster, the maximum density will be higher. In addition, the addition of the control variables $u$ and $v$ obviously has more remarkable effect on the density of rumor spreader and the density of true information spreader. This reveals the role of control variables in rumor propagating, which conforms to the above theoretical analysis. This picture provides practical advice on controlling the spread of rumors in reality. First of all, news media should carry out science programs through various channels in their daily life, spreading scientific knowledge, enhancing the scientific literacy of the public, and enhancing their judgment. Secondly, when rumors appear, the authorities should carry out activities to refute rumors in a timely manner and publicize the true information about the rumors. The combination of these two 


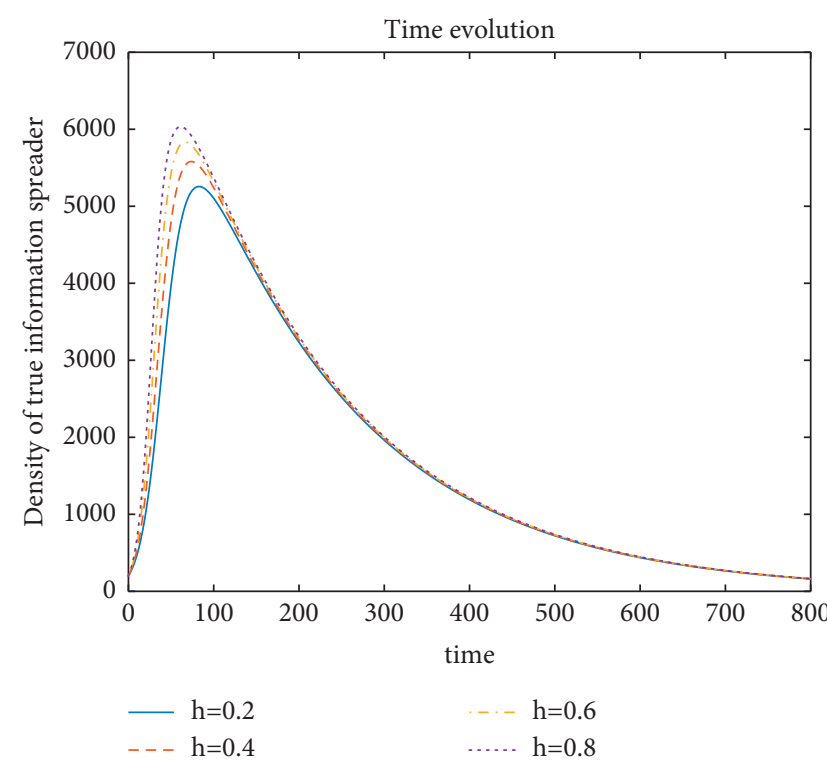

(a)

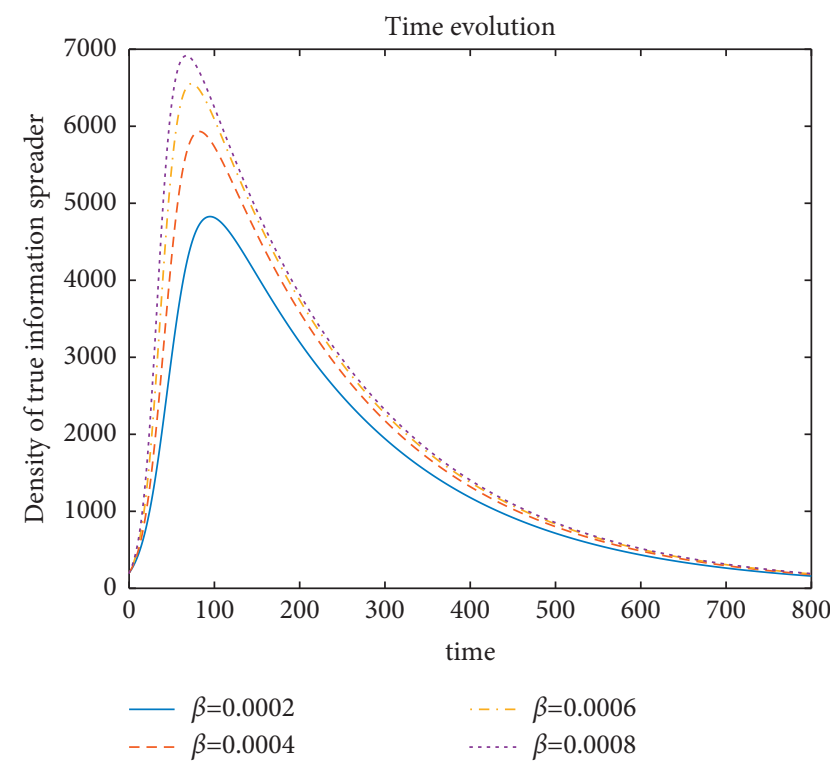

(b)

FIgURE 4: The impact of different parameters $h, \beta$ on the density of true information spreader without control.
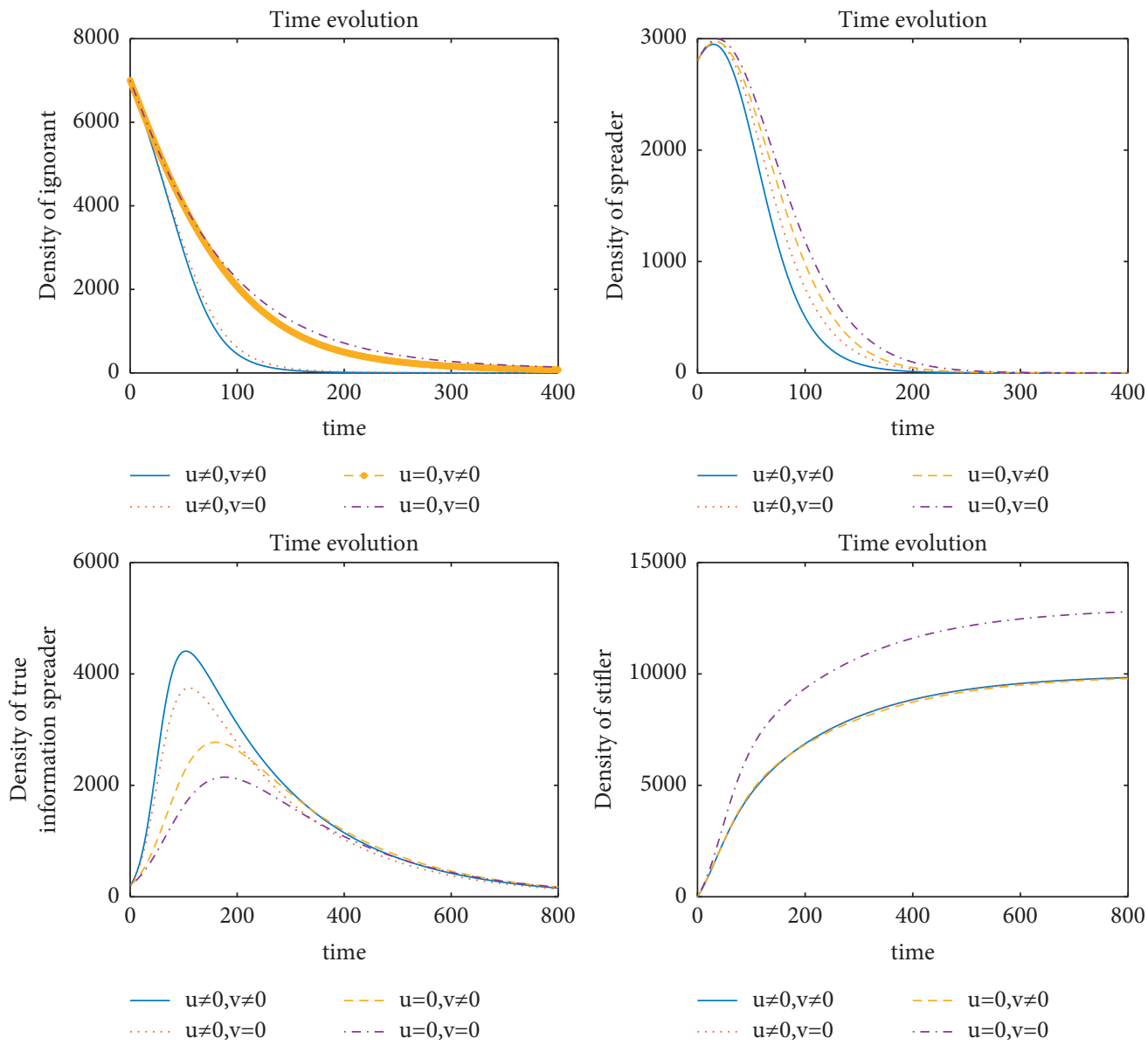

FIgURE 5: Time evolution of density of different group with control or without control. 
methods can effectively control rumor propagating and lower the harm of rumors.

\section{Conclusions}

In terms of studying the dynamics of rumor propagating, this article mainly considers the role of true information spreader and social reinforcement of these factors. Adding true information spreader to the model, we have established ISTR model, and we have studied the dynamics of rumor propagation under the effect of social reinforcement. In this process, we have taken advantage of the characteristics of heterogeneous networks and have adopted a mean field method to solve the transformation probability of an ignorant person turning into a rumor spreader or a true information spreader and have calculated the mean field equation of the model. Then, according to the mean field equation, we proved that the rumor propagation model has an equilibrium point and obtained the basic regeneration number of the rumors. The simulation experiment has proved that, after joining true information spreader, some ignorant people and rumor spreader will turn into true information spreader, so the density of rumor spreader will decrease, and the propagation speed of rumor slows down. At the same time, social reinforcement for rumor promotes the propagation of rumors, while social reinforcement for true information hinders the propagation of rumors. Then, the basic reproduction number of rumors has been proved in the model.

On the basis of dynamic analysis of rumor propagation, we have studied rumor control strategies. Then, we have added two kinds of control variables $u$ and $v$, established the rumor control model, obtained the optimal control strategies by using the optimal control theory, and obtained the optimal solution of the control variables by using Pontryagin's Minimum Principle of optimality, where the control variable $u$ represents the scientific knowledge popularization, and the control variable $v$ represents the rumor refutation strategies. Then the simulation part has showed the comparison diagram with and without control and compares the changes of the density of four groups under different condition. The results have proved that the control variable $u$ increases the ratio of ignorant people turning to true information spreaders. The control variable $v$ increases the ratio of rumor spreaders turning to true information spreaders. And the addition of control variables $u$ and $v$ has a greater impact on the rumor spreaders and the true information spreaders. This has proved that control variables can achieve the purpose of controlling rumors by reducing the amount of rumor spreaders and increasing the amount of true information spreaders.

\section{Data Availability}

The data used to support the findings of this study are included within the article.

\section{Conflicts of Interest}

The authors declare that they have no conflicts of interest.

\section{Acknowledgments}

This work was partially supported by the Program for Professor of Special Appointment (Eastern Scholar) at Shanghai Institutions of Higher Learning and the project for the Natural Science Foundation of Shanghai (21ZR1444100), the project for the National Natural Science Foundation of China (72174121, 71774111, 71871144, and 71804047), and the projects of the National Social Science Foundation of China (21BGL188).

\section{References}

[1] S. A. Alkhodair, S. H. Ding, B. C. Fung, and J. Liu, "Detecting breaking news rumors of emerging topics in social media," Information Processing \& Management, vol. 57, no. 2, Article ID 102018, 2020.

[2] Y. Cui, S. Ni, S. Shen, and Z. Wang, "Modeling the dynamics of information dissemination under disaster," Physica A: Statistical Mechanics and Its Applications, vol. 537, Article ID 122822, 2020.

[3] D. J. Daley, D. G. Kendall, Epidemics and rumors," Nature, vol. 204, p. 1188, 1965.

[4] D. P. Maki-Thompson, Mathematical Models and Applications, with Emphasis on Social, Life, and Management Science, Englewood Cliffs, New Jersey, United States, 1973.

[5] A. Sudbury, "The proportion of the population never hearing a rumour," Journal of Applied Probability, vol. 22, no. 2, pp. 443-446, 1985.

[6] R. Pastor-Satorras, C. Castellano, P. Van Mieghem, and A. Vespignani, "Epidemic processes in complex networks," Reviews of Modern Physics, vol. 87, no. 3, pp. 925-979, 2015.

[7] D. H. Zanette, "Critical behavior of propagation on smallworld networks," Physical review. E, Statistical, nonlinear, and soft matter physics, vol. 64, no. 5, Article ID 050901, 2001.

[8] Y. Moreno, M. Nekovee, and A. F. Pacheco, "Dynamics of rumor propagation in complex networks," Physical Review, vol. 69, no. 6, Article ID 066130, 2004.

[9] H. Sun, Y. Sheng, and Q. Cui, "An uncertain SIR rumor spreading model," Advances in Difference Equations, vol. 1, pp. 1-22, 2021.

[10] Y.-Q. Wang, X.-Y. Yang, Y.-L. Han, and X.-A. Wang, "Rumor spreading model with trust mechanism in complex social networks," Communications in Theoretical Physics, vol. 59, no. 4, pp. 510-516, 2013.

[11] L. Zhao, Q. Wang, J. Cheng, Y. Chen, J. Wang, and W. Huang, "Rumor spreading model with consideration of forgetting mechanism: a case of online blogging LiveJournal," Physica A: Statistical Mechanics and Its Applications, vol. 390, no. 13, pp. 2619-2625, 2011.

[12] M. Jiang, Q. Gao, and J. Zhuang, "Reciprocal spreading and debunking processes of online misinformation: a new rumor spreading-debunking model with a case study," Physica A: Statistical Mechanics and Its Applications, vol. 565, Article ID 125572, 2021.

[13] J. Wang, H. Jiang, C. Hu, Z. Yu, and J. Li, "Stability and Hopf bifurcation analysis of multi-lingual rumor spreading model with nonlinear inhibition mechanism," Chaos, Solitons \& Fractals, vol. 153, Article ID 111464, 2021.

[14] S. Yu, Z. Yu, H. Jiang, and S. Yang, "The dynamics and control of 2I2SR rumor spreading models in multilingual online social networks," Information Sciences, vol. 581, pp. 18-41, 2021. 
[15] L. Zhu, G. Guan, and Y. Li, "Nonlinear dynamical analysis and control strategies of a network-based SIS epidemic model with time delay," Applied Mathematical Modelling, vol. 70, pp. 512-531, 2019.

[16] J. Wang, H. Jiang, T. Ma, and C. Hu, "Global dynamics of the multi-lingual SIR rumor spreading model with cross-transmitted mechanism," Chaos, Solitons \& Fractals, vol. 126, pp. 148-157, 2019.

[17] Y. Zhou, C. Wu, Q. Zhu, Y. Xiang, and S. W. Loke, "Rumor source detection in networks based on the SEIR model," IEEE Access, vol. 7, pp. 45240-45258, 2019.

[18] A. Yang, X. Huang, X. Cai, X. Zhu, and L. Lu, "ILSR rumor spreading model with degree in complex network," Physica A: Statistical Mechanics and Its Applications, vol. 531, Article ID 121807, 2019.

[19] G. Y. Jiang, S. P. Li, and M. L. Li, "Dynamic rumor propagation of public opinion reversal on Weibo based on a twostage SPNR model," Physica A: Statistical Mechanics and Its Applications, vol. 558, Article ID 125005, 2020.

[20] L. Zhu and B. Wang, "Stability analysis of a SAIR rumor spreading model with control strategies in online social networks," Information Sciences, vol. 526, pp. 1-19, 2020.

[21] K. M. A. Kabir, K. Kuga, and J. Tanimoto, "Analysis of SIR epidemic model with information spreading of awareness," Chaos, Solitons \& Fractals, vol. 119, pp. 118-125, 2019.

[22] B. Karrer and M. E. Newman, "Message passing approach for general epidemic models," Physical review. E, Statistical, nonlinear, and soft matter physics, vol. 82, no. 1, Article ID 016101, 2010.

[23] F. Wang, Z. Zhu, and P. Liu, "Influence maximization in social network considering memory effect and social reinforcement effect," Future Internet, vol. 11, no. 4, pp. 1-16, 2019.

[24] D. A. Vega-Oliveros, L. Da Fontoura Costa, and F. A. Rodrigues, "Influence maximization by rumor propagation on correlated networks through community identification," Communications in Nonlinear Science and Numerical Simulation, vol. 83, Article ID 105094, 2020.

[25] C. Liu, X. X. Zhan, Z. K. Zhang, G. Q. Sun, and P. K. Hui, "How events determine spreading patterns: information transmission via internal and external influences on social networks," New Journal of Physics, vol. 17, no. 11, Article ID $113045,2015$.

[26] Y. Xu, R. Mei, Y. Yang, and Z. Kong, "Modeling and analysis of rumor propagation with social reinforcement mechanism," Advances in Mathematical Physics, vol. 2019, Article ID 7241021, 2019.

[27] L. A. Huo and S. J. Chen, "Rumor propagation model with consideration of scientific knowledge level and social reinforcement in heterogeneous network," Physica A: Statistical Mechanics and Its Applications, vol. 559, Article ID 125063, 2020.

[28] S. Vosoughi, D. Roy, and S. Aral, "The spread of true and false news online," Science, vol. 359, no. 6380, pp. 1146-1151, 2018.

[29] L. Yang, Z. Li, and A. Giua, "Rumor containment by spreading correct information in social networks," in Proceedings of the 2019 American Control Conference (ACC), pp. 5608-5613, IEEE, Philadelphia, PA, USA, July 2019.

[30] M. Koohikamali and D. J. Kim, "Rumor and truth spreading patterns on social network sites during social crisis: big data analytics approach," Lecture Notes in Business Information Processing in Proceedings of the Workshop on E-Business, vol. 258, pp. 166-170, Dublin, Ireland, September 2016.

[31] L. Zhao, X. Wang, J. Wang, X. Qiu, and W. Xie, "Rumorpropagation model with consideration of refutation mechanism in homogeneous social networks," Discrete Dynamics in Nature and Society, vol. 2014, Article ID 659273, 11 pages, 2014.

[32] X.-X. Zhao and J.-Z. Wang, "Dynamical behaviors of rumor spreading model with control measures," Abstract and Applied Analysis, vol. 2014, pp. 1-11, Article ID 247359, 2014.

[33] M. Askarizadeh, B. Tork Ladani, and M. H. Manshaei, "An evolutionary game model for analysis of rumor propagation and control in social networks," Physica A: Statistical Mechanics and Its Applications, vol. 523, pp. 21-39, 2019.

[34] G. Chen, "ILSCR rumor spreading model to discuss the control of rumor spreading in emergency," Physica A: Statistical Mechanics and Its Applications, vol. 522, pp. 88-97, 2019.

[35] M. Ahsan, M. Kumari, and T. P. Sharma, "Rumors detection, verification and controlling mechanisms in online social networks: a survey," Online Social Networks and Media, vol. 14, pp. 1-12, 2019.

[36] L. A. Huo, L. Wang, and X. Zhao, "Stability analysis and optimal control of a rumor spreading model with media report," Physica A: Statistical Mechanics and Its Applications, vol. 517, pp. 551-562, 2019. 